\title{
CONHECIMENTO DE ENFERMEIROS DE UNIDADES DE ATENÇÃO BÁSICA ACERCA DA TUBERCULOSE
}

Reinaldo Antonio Silva Sobrinho ${ }^{1}$ Angélica Liliane de Souza ${ }^{2}$, Anneliese Domingues Wysocki Laís Mara Caetano da Silva ${ }^{4}$, Aline Ale Beraldo ${ }^{3}$, Tereza Cristina Scatena Villa ${ }^{5}$

RESUMO: O estudo quantitativo de corte transversal analisou o conhecimento de 30 enfermeiros da Atenção Básica à Saúde sobre a tuberculose no município de Foz do Iguaçu, por meio de questionário semiestruturado aplicado de março à abril de 2009. A maioria era do sexo feminino (93,3\%), atuava em Unidade de Saúde da Família (73,3\%) e relatou não ter recebido treinamento em tuberculose (66,7\%). A média de anos trabalhados na Atenção Básica à Saúde foi de 4,3 (DP $=3,7$ anos). Verificou-se que o conhecimento dos profissionais em relação à prevenção, transmissão, diagnóstico e tratamento da tuberculose foram superficiais, resultados que apontam para a necessidade de organização de capacitações na área. DESCRITORES: Tuberculose; Enfermagem; Atenção primária à saúde.

\section{CONOCIMIENTO DE ENFERMEROS DE UNIDADES DE ATENCIÓN BÁSICA ACERCA DE LA TUBERCULOSIS}

RESUMEN: El estudio cuantitativo transversal analizó el conocimiento de 30 enfermeros de la Atención Básica a la Salud sobre la tuberculosis en el municipio de Foz do Iguaçu, por medio de cuestionario semiestructurado aplicado de marzo a abril de 2009. La mayoría era del sexo femenino (93,3\%), actuaba en Unidad de Salud de la Familia (73,3\%) y relató no haber hecho entrenamiento en tuberculosis (66,7\%). La media de años trabajados en la Atención Básica a la Salud fue de 4,3 (DP = 3,7 anos). Se ha verificado que los conocimientos de los profesionales acerca de la prevención, transmisión, diagnóstico y tratamiento de la tuberculosis fueron superficiales, resultados que apuntan para la necesidad de organización de capacitaciones en el área.

DESCRITORES: Tuberculosis; Enfermería; Atención primaria a la salud.

\section{PRIMARY HEALTH CARE CENTER NURSES' KNOWLEDGE REGARDING TUBERCULOSIS}

\begin{abstract}
This quantitative cross-sectional study analysed the knowledge of 30 primary care nurses regarding tuberculosis in the city of Foz do Iguaçu, using a semi-structured questionnaire applied in March and April 2009. The majority were female (93.3\%), worked in a Family Health Center (73.3\%) and reported not having received training in tuberculosis $(66.7 \%)$. The mean number of years worked in primary care was 4.3 (SD = 3.7 years). It was ascertained that the professionals' knowledge relating to the prevention, transmission, diagnosis and treatment of tuberculosis was superficial; these results indicate the need to organize training in this area.
\end{abstract}

DESCRIPTORS: Tuberculosis; Nursing; Primary care.

\footnotetext{
${ }^{1}$ Enfermeiro. Doutor em Enfermagem. Professor da Universidade Estadual do Oeste do Paraná. Foz do Iguaçu-PR-Brasil ${ }^{2}$ Enfermeira.

${ }^{3}$ Enfermeira. Mestre em Ciências da Saúde. Doutoranda em Saúde. Universidade de São Paulo. Ribeirão Preto-SP-Brasil

${ }^{4}$ Enfermeira. Doutoranda em Enfermagem em Saúde Pública. Universidade de São Paulo. Ribeirão Preto-SP-Brasil

${ }^{5}$ Enfermeira. Doutora em Enfermagem. Professora da Universidade de São Paulo. Ribeirão Preto-SP-Brasil
} 


\section{INTRODUÇÃO}

A tuberculose (TB) se configura como importante problema de saúde pública e, apesar da implementação de estratégias para seu controle, ainda permanecem elevadas as taxas de incidência e mortalidade pela doença. Frente à aproximadamente 9 milhões de casos novos de TB no mundo e 1,7 milhões de óbitos/ano, o Brasil ocupa o $22^{\circ}$ lugar no ranking dos 22 países com maior incidência da $\mathrm{TB}$ e o $14^{\circ}$ lugar em número de casos com baciloscopia positiva ${ }^{(1)}$, o que coloca o país desfavoravelmente em posição de destaque.

No cenário brasileiro, após a declaração do estado de emergência da TB, no final dos anos de 1990, foram desencadeadas mudanças na estratégia de controle da doença, preconizadas pelo Programa Nacional de Controle da Tuberculose. Essa estratégia tem como objetivo central a redução da incidência, por meio da detecção e do tratamento precoce da doença. E, dessa forma, o diagnóstico eficiente se apresenta como crescente desafio, ao se considerar não só as dificuldades dos doentes em acessar os serviços de saúde, como também as questões organizacionais do próprio sistema de saúde ${ }^{(2)}$.

Ressalta-se que no contexto nacional as ações de controle da TB são de responsabilidade da Atenção Básica à Saúde (ABS), por meio da Norma Operacional da Assistência à Saúde (NOAS), na qual são definidas áreas de atuação estratégica mínima da Gestão Plena da Atenção Básica Ampliada. Desta forma, os serviços de ABS devem realizar a identificação dos sintomáticos respiratórios, diagnóstico, tratamento e acompanhamento dos doentes ${ }^{(3)}$. Além disso, as equipes da ABS devem ser compostas, minimamente, por médico, enfermeiro, agente comunitário de saúde e auxiliar de enfermagem ou técnico de enfermagem. As atribuições comuns aos integrantes são busca ativa, registro dos agravos de notificação compulsória e de outros agravos, e situações de importância local, incluindo o controle da $\mathrm{TB}^{(4)}$.

$\mathrm{A}$ inserção do enfermeiro na atenção à $\mathrm{TB}$ no Brasil, por sua vez, remonta a década de 1960 quando ocorreram readequações na forma de tratamento da doença e identificou-se a necessidade de incluir profissionais capacitados para ações de educação sanitária, medicação supervisionada, controle de comunicantes e articulação com o dispensário que, atualmente, relaciona-se à referência e contra-referência no decorrer do processo de diagnóstico e tratamento da doença ${ }^{(4)}$.

Apesar dessa reorganização são reconhecidas as dificuldades para o diagnóstico precoce, decorrentes da degradação dos serviços e do despreparo dos profissionais de saúde para identificar a doença ${ }^{(5)}$. Embora tenham sido definidas as competências do enfermeiro na atenção à saúde de usuários com a TB na ABS, há inúmeras barreiras assistenciais, tais como a deficiência numérica de recursos humanos, sobrecarga de funções, inadequada qualificação, e visão centralizada e fragmentada da organização das ações de controle da TB (dispostos geralmente em serviços de nível secundário de atenção). Estas e outras barreiras podem comprometer $\mathrm{o}$ acesso dos doentes às ações de controle da doença na $\mathrm{ABS}^{(4)}$.

Em razão dessas dificuldades, um dos eixos de atuação do Ministério da Saúde (MS) é o incentivo à sensibilização dos gestores e dos profissionais que integram o Sistema Único de Saúde com o intuito de incrementar o conhecimento e qualificar as ações de controle da doença ${ }^{(3)}$. Sendo assim, é imprescindível a qualificação gerencial, organizativa e técnico-assistencial dos profissionais que atuam no controle da doença, inclusive os enfermeiros ${ }^{(6)}$.

Diante da importância que representa o conhecimento dos profissionais sobre TB, principalmente no que concerne à importância da atuação dos enfermeiros na concepção das ações para seu controle, este estudo se baseia na seguinte questão norteadora: qual o conhecimento dos enfermeiros da ABS sobre a TB e as suas ações de controle? Deste modo, o estudo objetivou analisar o conhecimento de enfermeiros da $\mathrm{ABS}$ em relação à TB, no município de Foz do Iguaçu, Paraná - Brasil.

\section{MÉTODO}

Trata-se de um estudo de corte transversal de caráter quantitativo, do tipo inquérito, realizado em Foz do Iguaçu, Paraná - Brasil, no ano de 2009. Neste município, os serviços de saúde estão organizados em cinco Distritos Sanitários, dentre os quais estão distribuídas 11 Unidades Básicas de Saúde (UBS) e 16 Unidades de Saúde da Família (USF) com 32 Equipes de Saúde da Família (ESF) as quais oferecem cobertura de $38 \%$ da população. Em 2009, no que se refere à TB, o Estado do Paraná apresentou incidência de 23,2 casos/100.000 habitantes, enquanto o município obteve a taxa de 38,7 casos/100.000 habitantes. As ações de controle da TB estão centralizadas no Programa de Controle de Tuberculose, iniciadas por meio do processo de descentralização para a $\mathrm{ABS}^{(7)}$.

No período de coleta de dados, as unidades de ABS contavam com 40 enfermeiros alocados em 24 unida- 
des e concordaram em receber os entrevistadores após contatos prévios e agendamento via telefone. Durante a visita foi exposta a finalidade da pesquisa e, após a leitura e a assinatura do Termo de Consentimento Livre e Esclarecido procederam-se as entrevistas nas próprias unidades de saúde, com 30 enfermeiros. Os demais profissionais recusaram-se a participar da pesquisa.

Para a coleta dos dados utilizou-se questionário semiestruturado, construído com base em recomendações científicas e operacionais disponibilizado pelo $\mathrm{MS}^{(3)}$, contendo 20 perguntas sobre a formação e experiência profissional, conhecimento dos profissionais quanto a prevenção, transmissão, diagnóstico, Tratamento Diretamente Observado (TDO), descentralização e atraso no diagnóstico da TB. As perguntas foram organizadas para serem respondidas segundo escalas de classificações variadas, como dicotômicas e de múltipla escolha, com resposta única ou múltipla.

Para a análise dos dados utilizou-se da estatística descritiva, sendo as variáveis apresentadas por meio de medianas, quartis e desvio padrão. As respostas do questionário relacionadas ao conhecimento sobre a TB foram categorizadas como adequadas, inadequadas ou parcialmente adequadas, de acordo com o Manual Técnico para o Controle da Tuberculose ${ }^{(3)}$.

Atendendo a resolução do Conselho Nacional de Saúde sobre ética em pesquisa envolvendo seres humanos, o projeto foi submetido ao Comitê de Ética da Universidade Estadual do Oeste do Paraná, e aprovado pelo parecer n. 022/2009.

\section{RESULTADOS}

Do total dos enfermeiros entrevistados, a maioria $(93,3 \%)$ era do sexo feminino e $26,7 \%$ fizeram especialização relacionada à ABS; a média de anos trabalhados foi de 4,3 anos ( $\mathrm{DP}=3,7$ anos), variando de 0,33 a 18 anos. Quanto ao tipo de unidade de saúde $73,3 \%$ estavam alocados em USF (Tabela 1 ).

O quadro 1 apresenta os resultados relativos às questões específicas sobre a doença, evidenciando que entre os enfermeiros que responderam as questões específicas sobre TB, de forma incorreta ou parcialmente incorreta, $75 \%$ apresentavam tempo de atuação elevado frente aos que responderam corretamente. Os que receberam treinamento, inversamente ao esperado, obtiveram menor percentual de acertos. Além disso, evidenciou-se que a maioria dos participantes não apresentou conhecimento suficiente sobre as condições de transmissão da doença, sinais e sintomas para suspeita de TB.

Quanto ao tratamento, mais da metade dos profissionais respondeu de forma parcialmente correta à questão sobre as orientações fornecidas ao doente, ao passo que $60 \%$ desconhecem a participação do usuário na escolha do local para a realização do TDO. A maioria não tinha certeza dos procedimentos necessários para identificar a resolutividade do tratamento.

Os profissionais expressaram sua opinião sobre a descentralização das ações de controle da TB para ABS, a estratégia DOTS (Directly Observed Treatment Short Course) e o retardo no diagnóstico da doença, sendo identificada inconsistência expressiva frente ao conhecimento da temática.

Tabela 1 - Distribuição das informações gerais dos enfermeiros de unidades básicas de saúde. Foz do Iguaçu-PR-Brasil, 2009

\begin{tabular}{|c|c|c|c|}
\hline Variáveis & & $\mathbf{n}$ & $\%$ \\
\hline \multirow{2}{*}{ Sexo } & Feminino & 28 & 93,3 \\
\hline & Masculino & 2 & 6,7 \\
\hline \multirow{4}{*}{ Titulação } & Graduação & 18 & 60 \\
\hline & Especialização em Saúde Pública & 3 & 10 \\
\hline & Especialização em Saúde da Família & 5 & 16,7 \\
\hline & Especialização em outras áreas & 4 & 13,3 \\
\hline \multirow{3}{*}{$\begin{array}{l}\text { Tipo de unidade } \\
\text { que atua }\end{array}$} & Unidade Básica de Saúde & 5 & 16,7 \\
\hline & Unidade Básica de Saúde com Programa de Agente Comunitário de Saúde & 3 & 10 \\
\hline & Unidade de Saúde da Família & 22 & 73,3 \\
\hline & $<1$ ano & 2 & 6,7 \\
\hline & 1 a 2 anos & 10 & 33,3 \\
\hline Tempo de traba- & 3 a 4 anos & 9 & 30 \\
\hline lho na unidade de & 5 a 6 anos & 4 & 13,3 \\
\hline \multirow[t]{3}{*}{ saúde } & 8 a 9 anos & 1 & 3,3 \\
\hline & 10 a 13 anos & 2 & 6,7 \\
\hline & 14 a 18 anos & 2 & 6,7 \\
\hline TOTAL & & $\mathbf{3 0}$ & 100 \\
\hline
\end{tabular}

Cogitare Enferm. 2014 Jan/Mar; 19(1):34-40 
Quadro 1 - Frequência de respostas específicas de enfermeiros sobre tuberculose. Foz do Iguaçu-PR-Brasil, 2009

\begin{tabular}{|c|c|c|c|c|c|c|}
\hline \multirow{2}{*}{\multicolumn{2}{|c|}{$\begin{array}{l}\text { Conhecimento sobre } \\
\text { tuberculose }\end{array}$}} & \multicolumn{2}{|c|}{ Recebimento de treinamento } & \multicolumn{2}{|c|}{ Tempo de atuação na área } & \multirow{2}{*}{$\begin{array}{c}\text { Total } \\
(\mathrm{n}=\mathbf{3 0})\end{array}$} \\
\hline & & Sim $(n=10)$ & Não $(n=20)$ & Mediana & Quartis & \\
\hline \multirow{2}{*}{$\begin{array}{l}\text { Forma de trans- } \\
\text { missão }\end{array}$} & Correto & $8(38,1 \%)$ & $13(61,9 \%)$ & 3 & {$[2 ; 4]$} & $21(70 \%)$ \\
\hline & Incorreto & $2(22,2 \%)$ & $7(77,8 \%)$ & 4 & {$[4 ; 12]$} & $9(30 \%)$ \\
\hline \multirow{2}{*}{$\begin{array}{l}\text { Condição para } \\
\text { transmissão }\end{array}$} & Correto & $4(36,4 \%)$ & $7(63,6 \%)$ & 2 & {$[2 ; 4]$} & $11(36,75)$ \\
\hline & Incorreto & $6(31,6 \%)$ & $13(68,4 \%)$ & 4 & {$[3 ; 6]$} & $19(63,3 \%)$ \\
\hline \multirow{2}{*}{$\begin{array}{l}\text { Indicador de risco } \\
\text { na transmissão }\end{array}$} & Correto & $6(37,5 \%)$ & $10(62,5 \%)$ & 3,5 & {$[2 ; 6]$} & $16(53,3 \%)$ \\
\hline & \begin{tabular}{|l|l} 
Incorreto \\
\end{tabular} & $4(28,6 \%)$ & $10(71,4 \%)$ & 3 & {$[2 ; 4]$} & $14(46,7 \%)$ \\
\hline \multirow{2}{*}{$\begin{array}{l}\text { Sinais e sinto- } \\
\text { mas para sus- } \\
\text { peita }\end{array}$} & \begin{tabular}{|l}
$\begin{array}{l}\text { Parcialmente } \\
\text { correto }\end{array}$ \\
\end{tabular} & $8(32 \%)$ & $17(68 \%)$ & 4 & {$[4 ; 12]$} & $25(83,3 \%)$ \\
\hline & Correto & $2(40 \%)$ & $3(60 \%)$ & 3 & {$[2 ; 5]$} & $5(16,7 \%)$ \\
\hline \multirow{3}{*}{$\begin{array}{l}\text { Sinais e sinto- } \\
\text { mas para sus- } \\
\text { peita }\end{array}$} & Correto & $1(33,3 \%)$ & $2(66,7 \%)$ & 3 & {$[0,1 ; 4]$} & $3(10 \%)$ \\
\hline & Incorreto & $9(36,6 \%)$ & $17(65,4 \%)$ & 3 & {$[2 ; 6]$} & $26(86,7 \%)$ \\
\hline & Não sabe & 0 & $1(100 \%)$ & & & $1(3,3 \%)$ \\
\hline \multirow{2}{*}{$\begin{array}{l}\text { Orientações bá- } \\
\text { sicas ao doente }\end{array}$} & Correto & $6(45,2 \%)$ & $7(53,8 \%)$ & 2,5 & {$[2 ; 4]$} & $13(43,3 \%)$ \\
\hline & \begin{tabular}{|l|} 
Parcialmente \\
correto \\
\end{tabular} & $4(23,5 \%)$ & $13(76,5 \%)$ & 4 & {$[2,5 ; 7,5]$} & $17(56,7 \%)$ \\
\hline \multirow{2}{*}{$\begin{array}{l}\text { Escolha do local } \\
\text { para tratamento }\end{array}$} & Correto & $4(33,3 \%)$ & $8(66,7 \%)$ & 4 & {$[2 ; 5,5]$} & $12(40 \%)$ \\
\hline & Incorreto & $6(33,3 \%)$ & $12(66,7 \%)$ & 3 & {$[2 ; 6]$} & $18(60 \%)$ \\
\hline \multirow{3}{*}{$\begin{array}{l}\text { Prioridade para } \\
\text { o tratamento }\end{array}$} & Correto & $6(46,2 \%)$ & $7(53,8 \%)$ & 3 & {$[2 ; 4]$} & $13(43,3 \%)$ \\
\hline & $\begin{array}{l}\text { Parcialmente } \\
\text { correto }\end{array}$ & $3(18,7 \%)$ & $13(81,3 \%)$ & 4 & {$[2 ; 6]$} & $16(53,4 \%)$ \\
\hline & Incorreto & $1(100 \%)$ & 0 & 3,5 & {$[3 ; 4]$} & $1(3,3 \%)$ \\
\hline \multirow{3}{*}{$\begin{array}{l}\text { Evidência para } \\
\text { identificação da } \\
\text { cura }\end{array}$} & Correto & $2(100 \%)$ & 0 & 3 & {$[2 ; 6]$} & $26,7 \%)$ \\
\hline & $\begin{array}{l}\text { Parcialmente } \\
\text { correto }\end{array}$ & $7(25,9 \%)$ & $20(74,1 \%)$ & 12 & - & $27(90 \%)$ \\
\hline & Incorreto & $1(100 \%)$ & 0 & 2,5 & {$[2 ; 4]$} & $1(3,3 \%)$ \\
\hline \multirow{2}{*}{$\begin{array}{l}\text { Identificação do } \\
\text { agravamento do } \\
\text { caso }\end{array}$} & $\begin{array}{l}\text { Parcialmente } \\
\text { correto }\end{array}$ & $4(40 \%)$ & $6(60 \%)$ & 4 & {$[2 ; 6]$} & $10(33,3 \%)$ \\
\hline & \begin{tabular}{|l|} 
Incorreto \\
\end{tabular} & $6(30 \%)$ & $14(70 \%)$ & 4 & {$[2 ; 5]$} & $20(66,7 \%)$ \\
\hline \multirow{2}{*}{$\begin{array}{l}\text { Medidas } \\
\text { preventivas }\end{array}$} & $\begin{array}{l}\text { Parcialmente } \\
\text { correto }\end{array}$ & $7(36,8 \%)$ & $12(63,2 \%)$ & 3 & {$[2 ; 13]$} & $19(63,3 \%)$ \\
\hline & \begin{tabular}{|l} 
Incorreto \\
\end{tabular} & $3(27,3 \%)$ & $8(72,7 \%)$ & 4 & {$[2 ; 6]$} & $11(36,7 \%)$ \\
\hline \multirow{3}{*}{$\begin{array}{l}\text { Descentraliza- } \\
\text { ção do diagnós- } \\
\text { tico e tratamento } \\
\text { para a Atenção } \\
\text { Básica }\end{array}$} & Bom & $6(25 \%)$ & $18(75 \%)$ & 2 & {$[2 ; 6]$} & $24(80 \%)$ \\
\hline & \begin{tabular}{|l|} 
Ótimo \\
\end{tabular} & $3(60 \%)$ & $2(40 \%)$ & 3 & - & $5(16,7 \%)$ \\
\hline & Não concorda & $1(100 \%)$ & 0 & 3 & {$[2 ; 5]$} & $1(3,3 \%)$ \\
\hline \multirow{2}{*}{$\begin{array}{l}\text { Conhecimento } \\
\text { sobre a estraté- } \\
\text { gia DOTS* }\end{array}$} & Sim & $5(41,7 \%)$ & $7(58,3 \%)$ & 3,5 & {$[2 ; 6]$} & $12(40 \%)$ \\
\hline & Não & $5(27,8 \%)$ & $13(72,2 \%)$ & 3,5 & {$[2 ; 6]$} & $18(60 \%)$ \\
\hline \multirow{3}{*}{$\begin{array}{l}\text { Fatores relacio- } \\
\text { nados ao retardo } \\
\text { do diagnóstico }\end{array}$} & $\begin{array}{l}\text { Fatores relaciona- } \\
\text { dos ao doente }\end{array}$ & $5(35,7 \%)$ & $9(64,3 \%)$ & 3 & {$[2 ; 4]$} & $14(46,7 \%)$ \\
\hline & $\begin{array}{l}\text { Fatores relacio- } \\
\text { nados aos ser- } \\
\text { viços de saúde }\end{array}$ & $5(33,3 \%)$ & $10(66,7 \%)$ & 4 & - & $15(50 \%)$ \\
\hline & Não sabe & 0 & $1(100 \%)$ & 2,5 & {$[2 ; 3]$} & $1(3,3 \%)$ \\
\hline Total & & $10(33,3 \%)$ & $20(66,7 \%)$ & 4 & {$[2 ; 6]$} & $30(100 \%)$ \\
\hline
\end{tabular}




\section{DISCUSSÃO}

Em 2004 o MS ratificou a política de saúde focada na ABS e, desde então, normatizou o controle da TB como responsabilidade dos municípios brasileiros e as ações de seu controle como de competência da ABS. Especificamente, cabe à ESF ações como vigilância em saúde, formação de equipes multiprofissionais, adscrição da população e longitudinalidade da atenção, necessárias para fortalecer os vínculos de responsabilidade e confiança entre os profissionais e a comunidade adstrita ${ }^{(6)}$. Os municípios buscam a descentralização das ações de controle da TB para a ABS, e é de grande importância o estudo da implantação desse plano estratégico também no município de Foz do Iguaçu, tendo em vista que foi possível identificar lacunas em um dos componentes fundamentais na estrutura dos serviços de saúde - o conhecimento dos profissionais sobre a TB; pequena parte dos enfermeiros pesquisados possuía curso de especialização em Saúde Pública ou Saúde da Família.

O despreparo da força de trabalho na ABS, quanto às atividades de controle da doença, foi verificada também no interior do Estado de São Paulo ${ }^{(6)}$. Estudo realizado em Minas Gerais ${ }^{(8)}$ mostrou que os trabalhadores com especialização possuem melhor desempenho na avaliação dos atributos da $\mathrm{ABS}$ e maior efetividade no desempenho diário das complexas funções neste ponto de assistência a saúde.

Ressalta-se que a formação de recursos humanos deve apresentar qualidade que assegure ao enfermeiro elevada competência para as ações assistenciais, principalmente no que concerne ao âmbito técnico-científico, administrativo e político, para que então ocorra transformação na prática sanitária ${ }^{(9)}$. Um dos elementos que podem colaborar para a melhoria desse quadro é a formação complementar, tendo em vista que o ensino da temática da TB nos cursos de graduação em saúde é precário ${ }^{(10)}$. Por conseguinte, a especialização ou residência em Saúde da Família pode ser opção para preparar os enfermeiros atuarem na ABS, com foco no processo saúde-doença e na organização de seu próprio processo de trabalho, bem como da equipe com a qual atua em conjunto.

Para alcançar o sucesso na implantação das ações de controle da TB são necessários arranjos que potencializem as ações de diagnóstico precoce e tratamento da doença. $\mathrm{O}$ fato de grande parte dos profissionais entrevistados apresentar dúvidas quanto à forma de transmissão da TB é preocupante e revela fragilidade nas atividades de diagnóstico precoce da doença em
Foz do Iguaçu, resultado também encontrado em uma pesquisa conduzida em outro município brasileiro ${ }^{(11)}$. Tal realidade não é encontrada somente no contexto nacional ${ }^{(12)}$. A falta de conhecimento sobre a causa, bem como o modo de transmissão da TB, torna deficiente o cuidado aos doentes e seus contatos, fato que potencializa o risco de transmissão da doença entre os profissionais e na população geral ${ }^{(13)}$.

A análise feita neste estudo sugere que os enfermeiros apresentaram dificuldades para a execução de ações de busca de sintomáticos respiratórios, uma vez que a baixa frequência de respostas corretas sobre a temática pode evidenciar despreparo para detectar os sinais e sintomas da TB. Destaca-se que somente 16,7\% dos entrevistados apontaram de forma correta os sinais clássicos da doença como possibilidade para suspeição, o que revela conhecimento limitado sobre o diagnóstico clínico. Estes resultados levantam dúvidas quanto à efetividade dos serviços de $\mathrm{ABS}$ no diagnóstico da doença, visto que essa deficiência colabora para o diagnóstico tardio, postergado para os serviços especializados e para o ambiente hospitalar, quando o quadro clínico da doença já está agravado, hipótese corroborada por um estudo no qual $50 \%$ dos casos tiveram o diagnóstico realizado em pronto socorro/hospital ${ }^{(14)}$.

Conhecer os sinais e sintomas da doença é condição básica para a realização do diagnóstico, sendo necessário também para a identificação da eficácia terapêutica ou evidência de falência. Dessa maneira, o reconhecimento da clínica da doença mostra-se intrínseco à realização do diagnóstico precoce e condição sine qua non para o controle do agravo prevendo, para isso, a inserção de profissionais capacitados e comprometidos nos serviços de saúde ${ }^{(6,15)}$.

Quanto às orientações ao doente sobre o tratamento, houve o predomínio de respostas parcialmente corretas e incorretas; os temas abordados variaram desde os efeitos adversos dos medicamentos até a consequência do abandono do tratamento e o TDO. A análise mostrou que os enfermeiros possuem conhecimentos insuficientes, especialmente em relação às evidências para identificar o agravamento da doença durante o tratamento e reconhecer se houve indícios de cura após o tratamento.

No que se relaciona à escolha do local mais apropriado para a realização do tratamento, descartou-se a participação do doente no cuidado, realidade semelhante a outro estudo ${ }^{(11)}$. Face ao exposto, a pesquisa revela situação diferente da preconizada pelo MS, na qual o usuário tem o direito de participação na escolha do local em que receberá o tratamento ${ }^{(6)}$. 
A comunicação ampla, baseada em aspectos biopsicossociais, e não restrita ao enfoque biológico, representa um fator que contribui para o sucesso terapêutico, como pôde ser demonstrado em estudo que identificou a perspectiva do atendimento pautado apenas em aspectos relacionados aos medicamentos, efeitos colaterais e duração do tratamento e resultou em maior risco para a não adesão à terapia medicamentosa ${ }^{(16)}$. Estas considerações são de extrema importância para a adesão ao tratamento, uma vez que este é bem sucedido quando o sujeito assume seu papel como corresponsável pela própria saúde. Para tal, o usuário do serviço deve ter conhecimento sobre todos os aspectos relacionados ao cuidado e participar das decisões de forma inter-relacionada, competente e resolutiva, não pautada apenas na lógica curativista, reiterando a necessidade de ampliação dos aspectos relacionados ao cuidado. Ressalta-se, nessa lógica, que melhor preparo proporciona ao enfermeiro a oportunidade de reunir condições para assistir ao doente com TB adequadamente. Nesta pesquisa houve relatos sobre as dificuldades de prover atendimento a esses doentes quando existe falta de capacitação em serviço, realidade também identificada em Teresina - Piauí ${ }^{17)}$.

$\mathrm{O}$ fato de os entrevistados aprovarem a descentralização das ações de controle da TB para a ABS revela a potencialidade dessa estratégia para fortalecer o vínculo entre a equipe de saúde e o doente, e possibilita o acompanhamento de sua história de vida, contribuindo para melhores resultados no tratamento e elevação das taxas de cura. Entretanto, para que a descentralização ocorra, plenamente, é necessário que o enfermeiro esteja apto a proporcionar atenção que envolva reflexões críticas sobre o processo de adoecimento e suas implicações culturais, sociais, políticas e econômicas. Embora reconheçam a importância da descentralização, a maioria dos profissionais referiu não ter conhecimento sobre o DOTS, corroborando com resultados encontrado em estudo realizado no Irã ${ }^{(18)}$. Esta estratégia deve ser de pleno conhecimento dos enfermeiros, especialmente quando as ações de controle da doença encontram-se sob a responsabilidade da ABS, tendo em vista que se apresenta como um dos pilares à garantia da adesão do paciente ${ }^{(6,12)}$.

Em relação à causa de atraso no diagnóstico, as respostas ficaram divididas entre fatores ligados aos doentes e aos serviços de saúde; a informação sobre a doença não pode ser considerada como responsabilidade exclusiva de usuários ${ }^{(19)}$.

Observou-se que o treinamento em TB não foi determinante para o conhecimento adequado das questões relacionadas à doença, de modo que treinamentos pontuais podem não ser suficientes para fornecer conhecimento e habilidade aos profissionais ${ }^{(13)}$. Independentemente dos motivos de tais lacunas, existe a necessidade de mudança desse quadro, com ênfase no preparo dos profissionais inseridos na $\mathrm{ABS}^{(12)}$.

A falta de ações de capacitação e de formação complementar em saúde pública pode ter contribuído para que os enfermeiros apresentassem conhecimento superficial sobre TB, estratégia DOTS e a descentralização das ações. Assegura-se que profissionais participantes de atividades de educação permanente estão mais capacitados para atender os usuários ${ }^{(20)}$, sendo essa prática de grande importância para que enfermeiros e os demais profissionais estejam aptos para auxiliar o usuário no processo de diagnóstico, tratamento e cura, utilizando-se da estratégia DOTS ${ }^{(17-18)}$.

Não foi identificada influência do tempo de atuação profissional na ABS com a proporção de respostas corretas em relação ao conhecimento sobre a TB, diferentemente de outro estudo ${ }^{(11)}$. Acredita-se que esse resultado, em parte, se deve ao pouco contato dos trabalhadores com o doente, pois a maioria dos casos são diagnosticados e tratados no ambulatório do Programa de Controle da Tuberculose, constatação que pode ser relacionada com o deficiência de conhecimento entre enfermeiros de $\mathrm{ABS}^{(19)}$. Sabe-se que o diagnóstico da TB em serviços secundários e terciários é comum entre doentes que já apresentam complicações e, muitas vezes, com necessidade de internação hospitalar; deste modo, discute-se que a problemática envolve todos os níveis do sistema de saúde. Há necessidade de programas de educação permanente com enfoque na TB, junto aos profissionais da $\mathrm{ABS}$, haja vista que essa estratégia potencializa o aprendizado dos profissionais, por meio da reflexão relacionada à realidade vivenciada no cotidiano do trabalho ${ }^{(6,17)}$.

\section{CONSIDERAÇÕES FINAIS}

O presente trabalho identificou conhecimento insuficiente dos enfermeiros no que se relaciona à atenção à TB, quadro que caminha na contramão da estratégia de controle da doença, proposta por meio da descentralização das ações para ABS. Os resultados apontam para a necessidade de instituir um programa de educação permanente com o objetivo de qualificar os enfermeiros nos diferentes aspectos relacionados ao diagnóstico, tratamento e controle da TB, contribuindo para a descentralização das ações de atenção à TB e a implantação da estratégia DOTS. 
Considera-se relevante propiciar espaços para a discussão do tema entre gestores, trabalhadores e comunidade, com intuito de viabilizar estratégias com vistas a atribuir maior importância à $\mathrm{TB}$ como problema de saúde pública persistente.

Este estudo pode apresentar limitações devido à presença de variáveis extrínsecas e os resultados encontrados não devem ser generalizados para outras localidades, haja vista as especificidades do cenário.

\section{REFERÊNCIAS}

1. Ministério da Saúde (BR). Secretaria de Vigilância em Saúde. Boletim Epidemiológico - Especial Tuberculose. Brasília (DF); 2012. p.1-12.

2. Almeida Filho AJ, Montenegro HRA, Santos TCF. A nova ordem no combate à tuberculose no Brasil: implicações para a enfermagem. Rev Rene. 2009;10(1):114-23.

3. Ministério da Saúde(BR). Programa Nacional de Controle daTuberculose. ManualdeRecomendações parao Controle da Tuberculose no Brasil. Brasília (DF); 2011. 186 p.

4. Ministério da Saúde (BR). Secretaria de Assistência à Saúde. Programa Nacional de Melhoria do Acesso e da Qualidade da Atenção Básica (PMAQ). Portaria/ SAS no 576, de 19 de setembro de 2011. Brasília (DF); 2011 [acesso em 30 jul 2012]. Disponível: http://cnes.datasus.gov.br/info_legislacao.asp

5. Beraldo AA, Arakawa T, Pinto ESG, Andrade RLP, Wysocki AD, Silva-Sobrinho RA, et al. Atraso na busca por serviço de saúde para o diagnóstico da tuberculose em Ribeirão Preto(SP). Ciênc. saúde colet. 2012;17(11):3079-86.

6. Monroe AA, Gonzales RIC, Palha PF, Sassaki CM, Ruffino-Netto A, Vendramini SHF, et al. Envolvimento de equipes da Atenção Básica à Saúde no controle da tuberculose. Rev Esc Enferm USP. 2008;42(2):262-7.

7. Secretaria Municipal de Saúde de Foz do Iguaçu (PR). Dados dos Sistemas de Informação em Saúde [Internet]. Foz do Iguaçu; 2008 [acesso em 20 ago 2009]. Disponível: http:www. fozdoiguacu.pr.gov.br/Portal/Pagina.aspx? Id=200.

8. Leão CDA, Caldeira AP. Avaliação da associação entre qualificação de médicos e enfermeiros em atenção primária em saúde e qualidade da atenção. Ciênc. saúde colet. 2011;16(11):4415-23.

9. Almeida MCP. A formação do enfermeiro frente à reforma sanitária. Cad. Saúde Pública. 1986;2(4):505-10.
10. Lavor A, Menezes KT. Não existe mau paciente, só serviço ineficiente. Rev. Radis. 2008;(69):21-3.

11. Maciel ELN, Araújo KW, Giacomin SS, Jesus FA, Rodrigues PM, Dietze R. Conhecimento de enfermeiros e médicos acerca da tuberculose na estratégia saúde da familia: um estudo de corte transversal. Ciênc. saúde colet. 2009;14(5):1397-404.

12. Basnet R, Hinderaker SG, Enarson D, Malla P, Morkve O. Delay in the diagnosis of tuberculosis in Nepal. BMC Public Health. 2009;9(236):1-5.

13. Teixeira EG, Menzies D, Cunha AJLA, Luiz RR, Ruffino-Netto A, Scartozzoni MS et al. Knowledge and practices of medical students to prevent tuberculosis transmission in Rio de Janeiro, Brazil. Rev. Panam. Salud Públ. 2008;24(4):265-70.

14. Perrechi MCT, Ribeiro SA. Desfechos de tratamento de tuberculose em pacientes hospitalizados e não hospitalizados no município de São Paulo. J. bras. pneumol. 2011;37(6):783-90.

15. Lienhardt C, Cobelens FGJ. Operational research for improved tuberculosis control: the scope, the needs and the way forward. Int J Tuberc Lung Dis. 2011;15(1):6-13.

16. Harry JA, Finkenflüge HJM, Moller V, Nieboer AP. TB treatment initiation and adherence in a South African community influenced more by perceptions than by knowledge of tuberculosis. BMC Public Health 2010;10(72):1-8.

17. Santos TMMG, Nogueira LT, Arcêncio RA. Atuação de profissionais da Estratégia Saúde da Família no controle da tuberculose. Acta Paul. Enferm. [Internet]. 2012;25(6):954-61.

18. Charkazi AR, Kouchaki G, Soleymani MN, Gholizade AH. Medical interns' knowledge of tuberculosis and DOTS strategy in northern Islamic Republic of Iran. East Mediterr Health J. 2010;16(12):1251-6.

19. Silva-Sobrinho RA, Andrade RLS, Ponce MAZ, Wysocki AD, Brunello MEF, Scatena LM et al. Retardo no diagnóstico da tuberculose em município da tríplice fronteira Brasil, Paraguai e Argentina. Rev. Panam. Salud Públ. 2012;31(6):461-8.

20. Silva ERP, Cazola LHO, Cheade MFM, Pícoli RP. Atuação dos agentes comunitários de saúde na estratégia saúde da família. Cogitare enferm. [Internet] 2012;17(4) [acesso em 08 fev 2013]. Disponível: http://ojs.c3sl.ufpr. br/ojs2/index.php/cogitare/article/view/30359/19636 